\title{
The Fuzzy Optimal Solution of Fuzzy Transport Problems Using A New Fuzzy Least Cost Method
}

\author{
Farikhin \\ faculty of science and mathematics \\ Univ. of Diponegoro \\ Semarang, Indonesia \\ muhammad.92sam@gmail.com \\ Bayu Surarso \\ faculty of science and mathematics \\ Univ. of Diponegoro \\ Semarang, Indonesia \\ bayusurarso@yahoo.co.id
}

\author{
Muhammad Sam'an \\ faculty of science and mathematics \\ Univ. of Diponegoro \\ Semarang, Indonesia \\ farikhin.math.undip@gmail.com \\ Bambang Irwanto \\ faculty of science and mathematics \\ Univ. of Diponegoro \\ Semarang, Indonesia \\ b_irwanto@yahoo.co.id
}

\begin{abstract}
The fuzzy transport algorithms is used by researchers for finding optimal solution of Fuzzy Transport Problem (FTP), one of them is the Least Cost Method or LCM. The concept of LCM is to choose the least cost from the FTP table to be used as the base cell. If there is the same lowest cost, it is freely chosen. However, the free election will affect the value and optimal fuzzy solutions obtained. Therefore, the fuzzy LCM as new method is proposed by adding weights using Simple Additive Weighting or SAW technique and new total integral value so that there will be unequal least costs. To illustrate the new proposed method, the completion of numerical examples is given and the results are reviewed with the results of the existing method. The advantages of the new proposed method can improve the shortcomings of the existing methods, as well as relevant to solving fuzzy transport problems in real life for use by decision makers..
\end{abstract}

Index Terms-Ranking fuzzy numbers, Fuzzy transport problem, New fuzzy least cost method, New ranking method, SAW..

\section{INTRODUCTION}

Now days, the globalization era is caused global market competition to become highly competitive. The companies must have better planning in making the right decision making during distribution process. How and when the distribute products to customers, they want minimal transport costs with a quantities products not exceeded capacity production limits and still fulfilled the minimum demand quantities for each destination. In this case, model of Transport Problem (TP) as one of the mathematics implementation can provide a comprehensive framework in planning product distribution. $\mathrm{TP}$ can be used for various sectors, especially in industries like investment of production, location of factory, control of inventory, scheduling of job, and many others. Generally, the solution of transport problem is done by assuming transport costs, value of supply and demand as crisp parameters, but in many cases the decision maker has no correctly information about the parameter belonging to the TP. If the information is an incorrect, then it lacks uncertainly, the corresponding parameters defining the problem can be formulated using fuzzy parameters or fuzzy numbers, giving rise to Fuzzy Transport Problems (FTP).

Basically, the TP is a problem of linear programming, simplied method is to implement the existing Fuzzy Linear Programming (FLP) approach to the FTP [1]-[4]. However, several of these approaches only give crisp solutions which represent a compromise solution in terms of fuzzy data. Actually, the solution of FTP has been discussed by several researchers. Gani and Razak [5] proposed a two-stage method to obtain minimum fuzzy costs on FTP with the quantities of supplies and demands in the form of fuzzy trapezoid. Dinagar and Palanivel [6] proposed the solving of FTP with a transport algorithm to determine the Initial Basic Feasible Solution (IBFS) and Modified Distribution (MODI) method to obtain optimal fuzzy solution is fuzzy trapezoid number. Pandian and Natarajan [7] introduced Zero point method to find IBFS and fuzzy optimal value of FTP with parameters fuzzy trapezoid numbers are transport costs per unit, quantities of supplies and demands. Chakraborty and Chakraborty [8] presented a time-cost minimization method to determine fuzzy optimal solution in which also the demands, supplies and transport cost per unit of the quantities are triangular fuzzy numbers. Basirzadeh [9] used the least cost method of conventional fuzzy transport algorithm for finding fuzzy IBFS and the MODI method to obtain the fuzzy optimal solution with transport costs, demands and supplies are trapezoid fuzzy numbers. He also used the magnitude method as rangking method to rank fuzzy numbers and the results of ranking are used to run the least cost approach and the MODI method to solve the FTP. Kaur and Kumar [10], [11] developed a classic fuzzy transport algorithm called the generalized fuzzy transport algorithm, namely generalized on NWC, LC and VAM to determine the IBFS and generalized the fuzzy MODI method to determine the optimal fuzzy solution. They used the quantities of transport per unit costs, demands and supplies are non-normal fuzzy numbers and used ranking functions to rank fuzzy optimal solutions. Fegade, et al. [12] used robust ranking 
as ranking method to rank fuzzy parameters like transport costs, supplies and demands are represented by triangular fuzzy numbers and also presented Zerro suffiq method to determine fuzzy optimal solution of FTP. Mohanaselvi and Ganesan [13] presented a new approach fo finding fuzzy IBFS of FTP and the magnitude ranking method to rank triangular fuzzy numbers that represented transport costs, supplies and demands. Samuel and Venkatachalapathy [14] proposed a new dual based method to find fuzzy optimal solution and using rangking function as ranking method to rank fuzzy transport costs that is represented triangular fuzzy numbers. Shanmugasundari and Ganesan [15] used VA method to obtain IBFS and MODI method to obtain optimal fuzzy solutions. They also use fuzzy trapezoid numbers to represent of transport costs, supplies and demands, but they did not use the ranking method for ranking or converting fuzzy numbers to a crisp numbers. Sudhagar and Ganesan [16] used the LC method to obtain IBFS and MODI methods to obtain optimal fuzzy solutions. They present numerical examples of FTP using fuzzy trapezoid numbers and also used the ranking score method to rank fuzzy numbers. Giarcarlo, et al., [17] proposed the Maximum Supply Method with Minimum Cost (MOMC) algorithm to determine fuzzy optimal solution and also used robust ranking technique as ranking method for the parameters fuzzy like transport costs, supply and demand are represented by trapezoid fuzzy numbers. Sam'an, et al. [18] used total integral ranking to rank fuzzy parameters of full FTP and Fuzzy Transport Algorithm (FTA) to determine fuzzy optimal solution. Sam'an, et al. [19] proposed the modified fuzzy transport algorithm to find fuzzy optimal solution with ranking score method to rank fuzzy parameters and also simple additive weighting to weight fuzzy cost.

Based on the method that has been proposed and discussed by the researchers above, many researchers used fuzzy transport algorithms for solving FTP, one of them is the Least Cost Method or LCM. The concept of LCM is to choose the least cost from the FTP table to be used as the base cell. If there is the same lowest cost, it is freely chosen. However, the free election will affect the value and optimal fuzzy solutions obtained. Therefore, the author presents the novelty of the fuzzy transport algorithm, namely the LCM modification using new total integral value as a ranking method or defuzzyfication of fuzzy numbers to crisp numbers and presents Simple Additive Weighting (SAW) as the weighting resulted of ranking fuzzy numbers. In addition, the results and discussion are presented with a numerical example as an illustration of the new proposed method implementation.

The paper is ordered as follows: Section 2 is the mathematical formulation of fuzzy transport problems. The ranking function of new total integral value is presented to to rank fuzzy numbers by section 3 . Then in section 4 , SAW technique is also presented to weight resulted of ranking fuzzy numbers. The new LCM is proposed to determine the value of fuzzy optimal solution from fuzzy transport problems by section 5 . Numerical example is presented in section 6 . The conclusion is discussed by section 7 .

\section{FuZZy TRANSPORT PROBlems Formulation}

The issue of transport optimization in theory, parameter supply $\left(\vartheta_{\imath}\right)$, demand $\left(\delta_{\jmath}\right)$ and transport cost $\left(\varsigma_{\imath \jmath}\right)$ are represented by crisp numbers is called crisp transport problem. However, if $\vartheta_{\imath}, \delta_{\jmath}$ and $\varsigma_{\imath \jmath}$ are represented by fuzzy numbers then crisp transport problem to be fuzzy transport problem, so that fuzzy parameters are notated $\widetilde{\vartheta_{\imath}}, \widetilde{\delta}_{j}$ and $\widetilde{\varsigma_{\imath \jmath}}$ Therefore, the formulation of fuzzy transport problem (FTP) as follows:

$$
\min \widetilde{T}
$$

subject $\mathrm{t}$

$$
\left\{\begin{array}{cc}
\sum_{j=1}^{m} \widetilde{\chi_{\iota \jmath}} \leq \widetilde{\vartheta_{\imath}} & \imath=1,2,3, \ldots, m \\
\sum_{\imath=1}^{n} \widetilde{\chi_{\iota \jmath}} \geq \widetilde{\delta_{\jmath}} & \jmath=1,2,3, \ldots, n \\
\widetilde{\chi_{\iota \jmath}} \geq \widetilde{0} & \forall \iota \jmath
\end{array}\right.
$$

where

$\min \widetilde{\mathrm{T}}=\sum_{\imath=1}^{m} \sum_{\jmath=1}^{n} \widetilde{\varsigma_{\imath \jmath}} \widetilde{\chi_{\iota \jmath}} \rightarrow$ total fuzzy transport cost

$\widetilde{\vartheta}_{\imath} \rightarrow$ fuzzy supplies numbers of product at $l^{\text {th }}$ source.

$\widetilde{\delta} \rightarrow$ fuzzy demands numbers of product at at $j^{\text {th }}$ destination

$\widetilde{\varsigma_{i j}} \rightarrow$ unit fuzzy transportation cost from $\iota^{t h}$ source to $j^{\text {th }}$ destination

$\widetilde{\chi_{\iota j}} \rightarrow$ approximate units of product numbers that should be delivered from $\iota^{\text {th }}$ source to $j^{\text {th }}$ destination.

Noted:

$\sum_{\imath=1}^{m} \widetilde{\vartheta}_{\imath}=\sum_{\jmath=1}^{n} \widetilde{\delta}_{\jmath}$ then FTP is named balanced FTP, if not it is called not balanced.

\section{New Total Integral RANKING}

New total integral value as the new ranking method that proposed as defuzzyfication or ordering fuzzy numbers that given by the non-normal fuzzy numbers to crisp number. The new ranking method is presented by the novel left, right, and integral values from inverse function of non- normal fuzzy numbers as follows:

Given $\widetilde{M}=(\alpha, \beta, \gamma, \eta: \varpi)$ is a non-normal trapezoidal fuzzy number. So, its membership function $\mu_{\widetilde{M}}$ of can be followed as

$$
\mu_{\widetilde{M}}(\chi)=\left\{\begin{array}{cc}
\varpi \frac{(\chi-\alpha)}{(\beta-\alpha)}, & \alpha \leq \chi<\beta \\
1, & \beta \leq \chi \leq \gamma \\
\varpi \frac{(\chi-\eta)}{(\gamma-\eta)}, & \gamma \leq \chi \leq \eta \\
0, & \text { otherwise }
\end{array}\right.
$$

It is obvious that if $L_{\widetilde{M}}=\varpi \frac{(\chi-\alpha)}{(\beta-\alpha)}$ with $L_{\widetilde{M}}$ : $[0, \varpi] \rightarrow[\alpha, \beta]$ and $R_{\widetilde{M}}=\varpi \frac{(\chi-\eta)}{(\gamma-\eta)}$ with $R_{\widetilde{M}}:[0, \varpi] \rightarrow[\gamma, \eta]$ then the inverse function of $L_{\widetilde{M}}$ and $R_{\widetilde{M}}$ can be denoted $\bar{L}_{\widetilde{M}}:[\alpha, \beta] \rightarrow[0, \varpi]$ and $\bar{R}_{\widetilde{M}}:[\gamma, \eta] \rightarrow[0, \varpi]$. Therefore, according Liou and Wang [20] $\bar{L}_{\widetilde{M}}$ and $\bar{R}_{\widetilde{M}}$ integrable on the closed interval $[0, \varpi]$. In the other word, $\bar{\mu}_{\widetilde{M}}=\int_{0}^{1} \bar{L}_{\widetilde{M}}(\varpi y) d y$ and $\bar{\mu}_{\widetilde{M}}=\int_{0}^{1} \bar{R}_{\widetilde{M}}(\varpi y) d y$ exist. Such that

$$
\text { where: }
$$

$$
\int_{0}^{1} \bar{L}_{\widetilde{M}}(\varpi y) d y=\left[\alpha+\frac{(\beta-\alpha) \varpi y}{\varpi}\right] d y=\frac{1}{2}(\alpha+\beta)
$$


Algorithm 1 Step 1. Determine $\bar{S}_{L}$ by (6)and $\bar{S}_{R}$ by (7), respectively; Step 2. Determine $\bar{S}_{J}$ by (8); Step 3. Find rank fuzzy numbers $\widetilde{M}_{1}$ and $\widetilde{M}_{2}$ with following properties: $(i)$ if $\bar{S}_{J}\left(\widetilde{M}_{1}\right)<\bar{S}_{J}\left(\widetilde{M}_{2}\right)$ the $\widetilde{M}_{1}<\widetilde{M}_{2} ;(i i)$ if $\bar{S}_{J}\left(\widetilde{M}_{1}\right)>$ $\bar{S}_{J}\left(\widetilde{M}_{2}\right)$ then $\widetilde{M}_{1}>\widetilde{M}_{2} ;($ iii $)$ if $\bar{S}_{J}\left(\widetilde{M}_{1}\right)=\left(\widetilde{M}_{2}\right)$ then $\widetilde{M}_{1}=\widetilde{M}_{2}$

and

$$
\int_{0}^{1} \bar{L}_{\widetilde{M}}(\varpi y) d y=\left[\eta+\frac{(\gamma-\eta) \varpi y}{\varpi}\right] d y=\frac{1}{2}(\gamma+\eta)
$$

considering from E.q (4) and (5), so the novel left and right integral value from inverse function of non-normal fuzzy numbers can be define

$$
\begin{aligned}
& \bar{S}_{L}\left(\widetilde{M)}=\varpi\left(\beta-\chi_{\min }\right)-\int_{0}^{1} \bar{L}_{\widetilde{M}}(\varpi y) d y\right. \\
& \Leftrightarrow S_{L}(\widetilde{M})=-\frac{\varpi}{2}(\alpha+3 \beta)-\chi_{\min }
\end{aligned}
$$

and

$$
\begin{aligned}
& \bar{S}_{R}\left(\widetilde{M)}=\varpi\left(\beta-\chi_{\min }\right)-\int_{0}^{1} \bar{R}_{\widetilde{M}}(\varpi y) d y\right. \\
& \Leftrightarrow \bar{S}_{R}(\widetilde{M})=-\frac{\varpi}{2}(3 \gamma+\eta)-\chi_{\min }
\end{aligned}
$$

where $\left.\chi_{\min }=\inf Q,{ }^{[}=1\right] 4 \bigcup=Q_{i}, Q_{i}=\left\{\frac{\mu_{\widetilde{M}}(\chi)}{\chi}>0\right\}$. Thus with $\lambda \in[0,1]$, by Eq. (7) and (8) the new total integral value of normal fuzzy numbers $\widetilde{M}$ can be obtained

$$
\begin{aligned}
& \bar{S}_{J}\left(\widetilde{M)}=\lambda \bar{S}_{R}\left(\widetilde{M)}+(1-\lambda) \bar{S}_{L}(\widetilde{M)}\right.\right. \\
& \Leftrightarrow \bar{S}_{J}\left(\widetilde{M)}=\left(\frac{\varpi}{2}\right)[(3 \gamma+\eta)-(1-\lambda)(\alpha+3 \beta)]-\chi_{\min }\right.
\end{aligned}
$$

Let $\widetilde{M}_{1}=\left(\alpha_{1}, \beta_{1}, \gamma_{1}, \eta_{1}: \varpi_{1}\right)$ and $\widetilde{M}_{2}=\left(\alpha_{2}, \beta_{2}, \gamma_{2}, \eta_{2}\right.$ : $\varpi_{2}$ ) be two generalized trapezoidal fuzzy numbers then use the following Algorithm 1 to compare $\widetilde{M}_{1}$ and $\widetilde{M}_{2}$.

\section{SAW METHOD}

Simple Additive Weighting (SAW) is the most widely used Multi-Attribute Decision Making techniques (MADM). It is very easy and the best of MADM techniques like WP, AHP, and TOPSIS which benefit from additive properties to determine the final score of an alternative or object. In SAW, the scores of each $j^{\text {th }}$ object are determined and they are ranked.

$$
\varphi_{\jmath}=\sum_{\jmath=1}^{n} \partial_{\imath} \ell_{\imath \jmath}
$$

where,

$\varphi_{\jmath} \rightarrow$ result weighting value of each $t^{t h}$ object.

$\partial_{\imath} \rightarrow$ initial weights on each $\imath^{\text {th }}$ source.

$\ell_{\imath \jmath} \rightarrow$ normalized performance rating of $j^{\text {th }}$ object on variabel $\imath^{t h}$ source $\imath=1,2, \ldots, m ; \jmath=1,2, \ldots, n$.

$\ell_{\imath \jmath}$ calculated by following formula:

- for profit variabel, we have:
Algorithm 2 Step 1. Determine the first weight for each each $\imath^{\text {th }}$ source $\left(\partial_{i}\right)$. the first weight is adapted to conditions in real life; Step 2. Determine $\ell_{\imath \jmath}$ by Eq (10), if $\widetilde{\varsigma_{\imath \jmath}}$ is variable of profit or Eq. (11), if e $\widetilde{\varsigma_{\imath \jmath}}$ is is variable of cost; Step 3. Determine last weight value of each $j^{\text {th }}$ object or $\varphi_{\jmath}$ using Eq (9) .

$$
\ell_{\imath \jmath}=\frac{\varsigma_{\imath \jmath}}{\max \varsigma_{\imath \jmath}},
$$

- for cost variabel, we have:

$$
\ell_{\imath \jmath}=\frac{\min \varsigma_{\imath \jmath}}{c_{i j}^{i}},
$$

with $0<\ell_{\imath \jmath} \leq 1, \imath=1,2, \ldots, m ; \jmath=1,2, \ldots, n$.

The algorithm in determining the final weight for each fuzzy transport cost $\left(\widetilde{\varsigma_{\imath \jmath}}\right)$ using SAW technique is given in Algorithm 2.

\section{The New Fuzzy Least Cost Method}

Several papers [9]-[19] have done to apply FTA of fuzzy Least Cost Method (LCM) for finding IBFS of FTP. However, it still has deficiencies in the estimation stage for maximum allocations $\widetilde{\chi_{\iota \jmath}}$ on cells $(\imath, \jmath)$ having the same $\widetilde{\varsigma_{\imath \jmath}}$ ranking. Therefore, The new fuzzy LCM as new proposed method have done obtaining $\widetilde{\varsigma_{\imath \jmath}}$ ranking is unequal. The new fuzzy LCM for finding IBFS is given Algorithm 3 and to find fuzzy optimal solution is given Algorithm 4.

Furthermore, we will be given about the existence of the new proposed algorithm.

Given the problem of fuzzy transportation problem is notated $\mathrm{FTP}^{1}$ as follows:

$$
\min \widetilde{\mathrm{T}}^{1}=\sum_{i=1}^{m} \sum_{\jmath=1}^{n} \widetilde{\varsigma_{\imath \jmath}} \widetilde{\chi_{\iota \jmath}}
$$

subject to

$$
\left\{\begin{array}{cc}
\sum_{j=1}^{m} \widetilde{\chi_{\iota \jmath}} \leq \widetilde{\vartheta_{\imath}} & \imath=1,2,3, \ldots, m \\
\sum_{\imath=1}^{n} \widetilde{\chi_{\iota \jmath}} \geq \widetilde{\delta_{\jmath}} & \jmath=1,2,3, \ldots, n \\
\widetilde{\chi_{\iota \jmath}} \geq 0 & \forall \iota \jmath
\end{array}\right.
$$

Next, given the problem of fuzzy transportation problem is notation FTP ${ }^{2}$ as follows:

$$
\min \widetilde{\mathrm{T}}^{2}=\sum_{\imath=1}^{m} \sum_{\jmath=1}^{n} \overline{S_{J}}\left(\widetilde{\varsigma_{\imath \jmath}}\right) \widetilde{\chi_{\iota \jmath}}
$$

subject to

$$
\left\{\begin{array}{cc}
\sum_{j=1}^{m} \widetilde{\chi_{\iota \jmath}} \leq \widetilde{\vartheta_{\imath}} & \imath=1,2,3, \ldots, m \\
\sum_{\imath=1}^{n} \widetilde{\chi_{\iota \jmath}} \geq \widetilde{\delta_{\jmath}} & \jmath=1,2,3, \ldots, n \\
\widetilde{\chi_{\iota \jmath}} \geq \widetilde{0} & \forall \iota \jmath
\end{array}\right.
$$

The existence of the the new proposed algorithm can be demonstrated by the following two theorems. 
Theorem 1. If $\left(\widetilde{\chi}_{11}^{\prime}, \widetilde{\chi}_{12}^{\prime}, \widetilde{\chi}_{13}^{\prime}, \ldots, \widetilde{\chi}_{\imath \jmath}^{\prime}, \ldots, \widetilde{\chi}_{m n}^{\prime}\right)$ is a fuzzy feasible solution to the FTP and and $\theta_{\imath \jmath}=\varsigma_{\imath \jmath}-\left(\kappa_{\imath}+\iota_{\jmath}\right) \geq$ $0 \forall \imath$, then $\left(\widetilde{\chi}_{11}^{\prime}, \widetilde{\chi}_{12}^{\prime}, \widetilde{\chi}_{13}^{\prime}, \ldots, \widetilde{\chi}_{\imath \jmath}^{\prime}, \ldots, \widetilde{\chi}_{m n}^{\prime}\right)$ is fuzzy optimal solution to the FTP ${ }^{2}$; where $\varsigma_{\imath \jmath}=\bar{S}_{J}\left(\widetilde{\varsigma_{\imath \jmath}}\right), \kappa_{\imath}$ and $\iota_{\jmath}$ are real numbers.

Proof: Given dual $\mathrm{FTP}^{2}$ is as follows:

$$
\max T^{2}=\sum_{\imath} \widetilde{\vartheta}_{\imath} \kappa_{\imath}+\sum_{\jmath} \widetilde{\delta}_{\jmath} \iota_{\jmath}
$$

subject to

$$
\left\{\kappa_{\imath}+\iota_{\jmath} \leq \overline{S_{J}}\left(\widetilde{\varsigma_{\imath \jmath}}\right)\right.
$$

where $\kappa_{\imath}$ and $\iota_{j}$ are unrestricted $\forall \imath \jmath$. Obvious that $\varsigma_{\imath \jmath}=$ $\overline{S_{J}}\left(\widetilde{\varsigma_{i j}}\right)$, so that constraints of dual FTP Eq. (16) and (17) becomes

$$
\begin{aligned}
& \kappa_{\imath}+\iota_{\jmath} \leq \overline{S_{J}}\left(\widetilde{\varsigma_{\imath \jmath}}\right) \\
& \Leftrightarrow \varsigma_{\imath \jmath}-\left(\kappa_{\imath}+\iota_{\jmath}\right) \geq 0 \\
& \Leftrightarrow \theta_{\imath \jmath} \geq 0
\end{aligned}
$$

Let ${\widetilde{\chi_{\imath \jmath}}}^{\prime}=\left(\widetilde{\chi}_{11}^{\prime}, \widetilde{\chi}_{12}^{\prime}, \widetilde{\chi}_{13}^{\prime}, \ldots, \widetilde{\chi}_{\imath \jmath}^{\prime}, \ldots, \widetilde{\chi}_{m n}^{\prime}\right)$ is fuzzy feasible solution of FTP $^{2}$ and $\theta_{\imath \jmath}=\varsigma_{\imath \jmath}-\left(\kappa_{\imath}+\iota_{\jmath}\right) \geq 0, \forall \imath \jmath$. We will points out that $\widetilde{\chi_{\imath \jmath}}$ is fuzzy optimal solution of FTP ${ }^{2}$ i.e by using the simplex method, value of $T_{\jmath}-\varsigma_{\jmath} \leq 0, \forall \jmath$ on FTP 2 obvious that

$$
\begin{aligned}
& \theta_{\imath \jmath}=\varsigma_{\imath \jmath}-\left(\kappa_{\imath}+\iota_{\jmath}\right) \geq 0, \forall \imath \jmath \\
& \Leftrightarrow-\theta_{\imath \jmath}=-\left[\varsigma_{\imath \jmath}-\left(\kappa_{\imath}+\iota_{\jmath}\right)\right] \leq 0 \\
& \Leftrightarrow-\theta_{\imath \jmath}=-\varsigma_{\imath \jmath}+\left(\kappa_{\imath}+\iota_{\jmath}\right) \leq 0 \\
& \Leftrightarrow \theta_{\imath \jmath}=\left(\kappa_{\imath}+\iota_{\jmath}\right)-\varsigma_{\imath \jmath} \geq 0
\end{aligned}
$$

Obvious that on the FTP ${ }^{2}$, value of $\left(\kappa_{\imath}+\iota_{\jmath}\right)-\varsigma_{\imath \jmath}=T_{\jmath}-\varsigma_{\jmath}$, so that obtained

$$
\theta_{\imath \jmath}=T_{\jmath}-\varsigma_{\jmath} \geq 0, \forall \imath \jmath
$$

because of $\widetilde{\chi_{\imath \jmath}}$ is fuzzy feasible solution of FTP ${ }^{2}$ and $\theta_{\imath \jmath}=$ $T_{\jmath}-\varsigma_{\jmath} \geq 0, \forall \imath \jmath$ on the table of the simplex method, so $\widetilde{\chi_{\imath \jmath}}$ is fuzzy optimal solution of FTP ${ }^{2}$.

Theorem 1 explained that arbitrary fuzzy optimal solution of FTP ${ }^{2}$ with $\theta_{\imath \jmath} \geq 0, \forall \imath \jmath$ is a fuzzy optimal solution of FTP $^{2}$. This theorem also guarantees that the determination of optimal criteria in step 4 on Algorithm 4 of the new proposed method is correct. Next, the optimal solution obtained in step 6 on Algorithm 4 of the new proposed method is fuzzy optimal value from $\mathrm{FTP}^{1}$ that can be guaranteed by the theorem 2 .

Theorem 2. If $\left(\widetilde{\chi}_{11}^{\prime}, \widetilde{\chi}_{12}^{\prime}, \widetilde{\chi}_{13}^{\prime}, \ldots, \widetilde{\chi}_{\imath \jmath}^{\prime}, \ldots, \widetilde{\chi}_{m n}^{\prime}\right)$ is fuzzy optimal solution of FTP ${ }^{2}$ then $\left(\widetilde{\chi}_{11}^{\prime}, \widetilde{\chi}_{12}^{\prime}, \widetilde{\chi}_{13}^{\prime}, \ldots, \widetilde{\chi}_{\imath \jmath}^{\prime}, \ldots, \widetilde{\chi}_{m n}^{\prime}\right)$ is fuzzy optimal solution of FTP ${ }^{1}$.

Proof: Let $\widetilde{\chi}_{\imath \jmath}^{\prime}=\left(\widetilde{\chi}_{11}^{\prime}, \widetilde{\chi}_{12}^{\prime}, \widetilde{\chi}_{13}^{\prime}, \ldots, \widetilde{\chi}_{\imath \jmath}^{\prime}, \ldots, \widetilde{\chi}_{m n}^{\prime}\right)$ is fuzzy optimal solution of FTP $^{2}$ and $\widetilde{\psi}_{\imath \jmath}^{\prime}=$ $\left(\widetilde{\psi}_{11}^{\prime}, \widetilde{\psi}_{12}^{\prime}, \widetilde{\psi}_{13}^{\prime}, \ldots, \widetilde{\psi}_{\imath \jmath}^{\prime}, \ldots, \widetilde{\psi}_{m n}^{\prime}\right)$ is fuzzy optimal solution of FTP ${ }^{1}$. If $\tilde{\chi}_{\imath \jmath}^{\prime}$ is fuzzy optimal solution of $\mathrm{FTP}^{2}$ then for every fuzzy feasible solution $\widetilde{\psi}_{\imath \jmath}^{\prime}$ is linear independent, such that obtained inequality

$$
\sum_{\imath=1}^{m} \sum_{\jmath=1}^{n} \overline{S_{J}}\left(\widetilde{\varsigma_{\imath \jmath}}\right){\widetilde{\chi_{\iota \jmath}}}^{\prime} \geq \sum_{\imath=1}^{m} \sum_{\jmath=1}^{n} \overline{S_{J}}\left(\widetilde{\varsigma_{\imath \jmath}}\right){\widetilde{\psi_{\iota \jmath}}}^{\prime}
$$

Similarly, if $\widetilde{\psi}_{\imath \jmath}^{\prime}$ is fuzzy optimal solution of $\mathrm{FTP}^{1}$ then for every fuzzy feasible solution $\widetilde{\chi}_{\imath \jmath}^{\prime}$ is linear independent, such that obtained inequality

$$
\sum_{\imath=1}^{m} \sum_{\jmath=1}^{n} \widetilde{\varsigma_{\imath \jmath}} \widetilde{\psi}_{\iota \jmath}{ }^{\prime} \leq \sum_{\imath=1}^{m} \sum_{\jmath=1}^{n} \widetilde{\varsigma_{\imath \jmath}} \widetilde{\chi} \iota \jmath^{\prime \prime}
$$

Finding rank of inequality (19) results that

$$
\begin{aligned}
& \overline{S_{J}}\left(\sum_{\imath=1}^{m} \sum_{\jmath=1}^{n} \widetilde{\varsigma_{\imath \jmath}} \widetilde{\psi_{\iota \jmath}}\right) \geq \overline{S_{J}}\left(\sum_{\imath=1}^{m} \sum_{\jmath=1}^{n} \widetilde{\varsigma_{\imath \jmath}} \widetilde{\chi_{\iota \jmath}}{ }^{\prime \prime \prime}\right) \\
& \Leftrightarrow \sum_{\imath=1}^{m} \sum_{\jmath=1}^{n} \overline{S_{J}}\left(\widetilde{\varsigma_{\imath \jmath}}\right) \widetilde{\chi_{\iota \jmath}} \geq \sum_{\imath=1}^{m} \sum_{\jmath=1}^{n} \overline{S_{J}}\left(\widetilde{\varsigma_{\imath \jmath}}\right){\widetilde{\psi_{\iota \jmath}}}^{\prime}
\end{aligned}
$$

Comparison of inequality (18) and (20) resulted

$$
\begin{aligned}
& \sum_{\imath=1}^{m} \sum_{\jmath=1}^{n} \overline{S_{J}}\left(\widetilde{\varsigma_{\imath \jmath}}\right) \widetilde{\chi_{\iota j}}=\sum_{\imath=1}^{m} \sum_{\jmath=1}^{n} \overline{S_{J}}\left(\widetilde{\varsigma_{\imath \jmath}}\right) \widetilde{\psi_{\iota \jmath}} \\
& \Leftrightarrow \sum_{\imath=1}^{m} \sum_{\jmath=1}^{n}{\widetilde{\chi_{\iota \jmath}}}^{\prime}=\sum_{\imath=1}^{m} \sum_{\jmath=1}^{n}{\widetilde{\psi_{\iota \jmath}}}^{\prime} \\
& \Leftrightarrow{\widetilde{\chi_{\iota \jmath}}}^{\prime}=\widetilde{\psi_{\iota \jmath}}
\end{aligned}
$$

So, it is proven if there is fuzzy optimal solution ${\widetilde{\chi_{\iota j}}}^{\prime}$ from FTP $^{2}$ then $\widetilde{\chi_{\iota j}}$ is fuzzy optimal solution from FTP $^{1}$

\section{NumericAl EXAMPLE}

To show a implementation of new proposed method on the FTP is given in Example 1 which is $\widetilde{\varsigma_{\imath j}}$ represented by nonnormal trapezoid fuzzy numbers, it is solved by using the new proposed methods and existing method.

Example 3. The numerical example on from Kaur and Kumar [11], fuzzy supplies approximate of the product available at three sources $\widehat{\vartheta}_{\imath}(\imath=1,2,3)$; fuzzy demands approximate of the product at three objects $\widetilde{\delta}_{\jmath}(\jmath=1,2,3)$ and fuzzy costs transport unit approximate of the product from each sources to each objects $\left(\widetilde{\varsigma_{\imath \jmath}}\right)$ are presented by non-normal trapezoid fuzzy number and given in Table 1. Calculate the fuzzy optimal solution such that the total fuzzy transport cost is minimum.

Solution 4. Clearly that $\sum_{\imath=1}^{m} \widetilde{\vartheta}_{\imath}=\sum_{\jmath=1}^{n} \widetilde{\delta}_{\jmath}=(9,17,23,30)$. So, Example 1 is said balanced FTP. Firstly, The converting of $\widetilde{\varsigma_{\imath \jmath}}$ to be crisp number $\varsigma_{\imath \jmath}$ is used by Algorithm 1 with $\partial_{1}=0.35, \partial_{2}=0.45$ and $\partial_{3}=0.2$. We compare the obtained result with existing method i.e Ebrahimnejad [21] that be seen Table 2.

From Table 2 be continued to obtain IBFS by using Algorithm 3. Then, to find fuzzy optimal solution by using Algorithm 4. We are compared the result that found by Kaur and Kumar [11] and Ebrahimnejad [21] that be seen Table 3 .

It should be noted as the fuzzy optimal solution and fuzzy optimal value that can be solved all methods proposed by Kaur and Kumar [11] and Ebrahimnejad [21]. These results of declared that if we want to solve a FTP based on ranking method, so that the new proposed method is more effective than the existing method. Reality, the depending on situation 
TABLE I

THE DATA OF THE FUZZY TRANSPORTATION PROBLEM

\begin{tabular}{|c|c|c|c|c|}
\hline & $\widetilde{\vartheta_{1}}$ & $\widetilde{\vartheta_{2}}$ & $\widetilde{\vartheta_{3}}$ & $\widetilde{\vartheta}_{\imath}$ \\
\hline \hline$\widetilde{\delta}_{1}$ & $(1,4,9,19 ; 0.5)$ & $(1,2,5,9 ; 0.4)$ & $(2,5,8,18 ; 0.5)$ & $(1,5,7,9 ; 0.2)$ \\
\hline$\widetilde{\delta_{2}}$ & $(8,9,12,26 ; 0.5)$ & $(3,5,8,12 ; 0.2)$ & $(7,9,13,28 ; 0.4)$ & $(4,7,8,10 ; 0.5)$ \\
\hline$\widetilde{\delta_{3}}$ & $(11,12,20,27 ; 0.5)$ & $(0,5,10,15 ; 0.8)$ & $(4,5,8,11 ; 0.6)$ & $(4,5,8,11 ; 0.6)$ \\
\hline$\widetilde{\delta_{j}}$ & $(3,5,8,12 ; 0.4)$ & $(4,8,9,10 ; 0.2)$ & $(2,4,6,8 ; 0.3)$ & \\
\hline
\end{tabular}

TABLE II

THE COMPARISON OF ORDERING FUZZY NUMBER

\begin{tabular}{|c|c|c|c|c|}
\hline Ranking method & \multicolumn{3}{|c|}{ Rank of $\varsigma_{\imath \jmath}$} & Ordering fuzzy numbers \\
\hline \multirow{3}{*}{ Existing method [21] } & 1.65 & 0.85 & 1.65 & \multirow{3}{*}{$\begin{aligned} \varsigma_{11} & =\varsigma_{13} \\
\varsigma_{22} & =\varsigma_{33}\end{aligned}$} \\
\hline & 2.75 & 1.4 & 2.85 & \\
\hline & 3.5 & 1.5 & 1.4 & \\
\hline \multirow{3}{*}{ New proposed method } & 1.3 & 1.22 & 1.26 & \multirow{3}{*}{ none } \\
\hline & 0.9 & 0.66 & 1.40 & \\
\hline & 1.4 & 2.44 & 0.87 & \\
\hline
\end{tabular}

TABLE III

THE COMPARISON OF $\widetilde{\chi_{\iota \jmath}}$ AND min $\widetilde{T}$

\begin{tabular}{|c|c|c|c|c|c|}
\hline Method finding of IBFS & \multicolumn{3}{|c|}{ Fuzzy solution optimal $\left(\widetilde{\chi_{\iota \jmath}}\right)$} & Fuzzy optimal value $(\min \widetilde{T})$ & Rank of min $\widetilde{T}$ \\
\hline Existing methods [11], [21] & $\begin{aligned} \widetilde{\chi_{11}} & =(0,0,1,2) \\
\widetilde{\chi_{21}} & =(1,3,3,4) \\
\widetilde{\chi_{31}} & =(2,2,3,5)\end{aligned}$ & $\begin{aligned} \widetilde{\chi_{12}} & =(1,4,5,6) \\
\widetilde{\chi_{22}} & =(3,4,4,4) \\
\widetilde{\chi_{23}} & =(0,0,0,0)\end{aligned}$ & $\begin{aligned} \widetilde{\chi_{13}} & =(0,1,1,1) \\
\widetilde{\chi_{23}} & =(0,0,0,0) \\
\widetilde{\chi_{33}} & =(2,3,5,7)\end{aligned}$ & $(48,99,222,500 ; 0.2)$ & 145.1 \\
\hline New proposed method & $\begin{aligned} \widetilde{\chi_{11}} & =(1,1,3,5) \\
\overline{\chi_{21}} & =(1,3,3,4) \\
\overline{\chi_{31}} & =(1,1,2,3)\end{aligned}$ & $\begin{aligned} \widetilde{\chi_{12}} & =(0,3,3,3) \\
\overline{\chi_{22}} & =(3,4,5,6) \\
\overline{\chi_{23}} & =(1,1,1,1)\end{aligned}$ & $\begin{aligned} \widetilde{\chi_{13}} & =(0,1,1,1) \\
\overline{\chi_{23}} & =(0,0,0,0) \\
\overline{\chi_{33}} & =(2,3,5,7)\end{aligned}$ & $(37,94,216,489 ; 0.2)$ & 144 \\
\hline
\end{tabular}

if the index of optimism is added to the ranking method for decision makers, the ranking method can make a unique solution or solution based on the level of significant. For example, assumed that the following new total integral value is used for the non-normal trapezoid fuzzy number $\widetilde{M}_{1}=$ $\left(\alpha_{1}, \beta_{1}, \gamma_{1}, \eta_{1}: \varpi_{1}\right)$ and $\widetilde{M}_{2}=\left(\alpha_{2}, \beta_{2}, \gamma_{2}, \eta_{2}: \varpi_{2}\right)$ by Eq. (8), where $\lambda \in[0,1]$ is the optimism index representing the optimism degree of a decision maker. If $\lambda=1$ is optimistic decision, $\lambda=0$ is pessimistic decision and $\lambda=0.5$ is moderate decision. Furthermore, the adding of weight to each $\widetilde{\varsigma_{\imath j}}$ also has an effect on the obtaining of ranking. The Simple Additive weighting (SAW) technique was considered very good at weighting. Based on Table 2, the existing method of result ranking $\widetilde{\varsigma_{\imath j}}$ for Examples 1 still obtain the same ranking value. These results can affect solutions and value of fuzzy optimal that be seen Table 3. Its table shows that the FTP is solved by using existing methods and the new proposed method results a different fuzzy optimal solution $\left(\widetilde{\chi_{\iota \jmath}}\right)$ and Fuzzy optimal value $(\min \widetilde{T})$. If the optimal fuzzy value is ranked using the new ranking method Eq. (8) then the optimal fuzzy value is obtained by the new proposed method is more minimal or smaller than the optimal value is obtained by the existing method. In other words, the new proposed method is better at doing ranking than the existing method.

Remark 5. The new proposed method i.e. the new fuzzy LCM can eliminate third case (if $\widetilde{\vartheta}_{\imath}=\widetilde{\delta}_{\text {j }}$ then either follow case 1 or case 2, but not both simultaneously Go to Step 2) to find $\widetilde{\chi_{\imath \jmath}}=\min \left(\widetilde{\vartheta}_{\imath}, \widetilde{\delta}_{\jmath}\right)$ on the classical method of LCM, such that to find $\widetilde{\chi_{\imath \jmath}}=\min \left(\widetilde{\vartheta}_{\imath}, \widetilde{\delta}_{\jmath}\right)$ only have 2 cases that be seen algorithm 3 of the new proposed method. Beside that, the new ranking method was proposed by adding SAW method to each ranking and the results has given a different ranking value.

\section{CONCLUSION}

This paper, a new fuzzy least cost method like as the one proposed to determine IBFS and the value of an fuzzy optimal solution FTP whose transport costs are represented as nonnormal fuzzy trapezoid numbers and products supplies and demands. The advantages of the new proposed method is presented using a numerical examples of FTP whose results can improve from the shortcomings of existing methods. The new proposed method is also very easy to understand, makes sense and can be used by decision makers to solve transport problems in real life.

\section{ACKNOWLEDGMENT}

The authors would like to thank two anonymous reviewers for their constructive comments and suggestions, which have helped to improve this paper. Furthermore, this research is supported by Grant of RISET MADYA FSM 2019 University of Diponegoro. 
Algorithm 3 Step 1. Formulate a mathematical model in real life of FTP as on the Eq. (1) and Eq. (2); Step 2. Make Table of FTP (FTPT) from a mathematical model of FTP that contained $\widetilde{\varsigma_{\imath j}}$ as fuzzy transport cost, $\widetilde{\vartheta}_{\imath}$ and $\widetilde{\delta}_{j}$ as fuzzy quantities of supplies and demands, respectively; Step 3. Check whether: $(i)$ if $\sum_{\imath=1}^{m} \widetilde{\vartheta}_{\imath}=\sum_{\jmath=1}^{n} \widetilde{\delta}_{\jmath}$ then next step 4; (ii) if $\sum_{\imath=1}^{m} \widetilde{\vartheta}_{\imath} \neq \sum_{\jmath=1}^{n} \widetilde{\delta}_{\jmath}$ then there are two conditions as follows: (a) $\sum_{\imath=1}^{m} \widetilde{\vartheta}_{\imath}>\sum_{\jmath=1}^{n} \widetilde{\delta}_{\jmath}$, enter a fuzzy factitious column that have all its $\widetilde{\varsigma_{\imath j}}$ as zero trapezoid fuzzy number or e $\widetilde{\varsigma_{\imath \jmath}}=(0,0,0,0)$ Let $\sum_{l=1}^{m} \widetilde{\vartheta}_{\imath}-\sum_{\jmath=1}^{n} \widetilde{\delta_{j}}$ as the fuzzy demand at this factitious object. Next step 4,(b) $\sum_{\imath=1}^{m} \widetilde{\vartheta}_{\imath}<\sum_{\jmath=1}^{n} \widetilde{\delta}_{\jmath}$, enter a fuzzy factitious row that have all its $\widetilde{\varsigma_{\imath \jmath}}$ as zero trapezoid fuzzy number or e $\widetilde{\varsigma_{\imath j}}=(0,0,0,0)$ Let $\sum_{\jmath=1}^{n} \widetilde{\delta_{\jmath}}-\sum_{\imath=1}^{m} \widetilde{\vartheta}_{\imath}$ as the fuzzy supply at this factitious object. Next step 4; Step 4. Converting $\widetilde{\varsigma_{\imath \jmath}}$ to be $\varsigma_{\imath \jmath}$ as crisp transport costs by using Algorithm 1; Step 5. Determine last weight value of each $j^{\text {th }}$ objects $\left(\varphi_{j}\right)$ by using Algorithm 2; Step 6 . Multiplying obtain $\varphi_{\text {, }}$ of Step 5 with crisp transport cost or $\varphi_{\jmath} \times \varsigma_{\imath \jmath}$; Step 7. Find the smallest $\varsigma_{\imath \jmath}$ in FTPT and determine minimum of $\widetilde{\vartheta}_{\imath}$ or $\widetilde{\delta}_{\jmath}$. Next, will come two possibilities are $(i)$ $\min \left(\widetilde{\vartheta}_{\imath}, \widetilde{\delta}_{\jmath}\right)=\widetilde{\vartheta}_{\imath}$, so make on $\widetilde{\chi_{\iota \jmath}}=\widetilde{\vartheta}_{\imath}$ where $m \times n$ FTPT of the new least cost. Then, neglect row of $\imath^{\text {th }}$ to find a new FTPT with $(m-1) \times n$ ordering. Change $\widetilde{\delta}_{\jmath}$ by e $\widetilde{\delta}_{\jmath} \ominus \widetilde{\vartheta}_{\imath}$ in the new FTPT. Next step 8 . (ii) $\min \left(\widetilde{\vartheta}_{\imath}, \widetilde{\delta}_{\jmath}\right)=\widetilde{\delta}_{\jmath}$, so make on $\mathrm{f} \widetilde{\chi_{\iota \jmath}}=\widetilde{\delta_{\jmath}}$ where $m \times n$ FTPT of the new least cost. Then, neglect column of $\widetilde{\jmath}^{\text {th }}$ to find a new FTPT with $m \times(n-1)$ ordering. Change $\widetilde{\delta_{j}}$ by $\widetilde{\vartheta}_{\imath}-\widetilde{\delta}_{\jmath}$ in the new FTPT. Next step 8; Step 8. Repeat Step 1 of the new FTPT, until is reduced to be a FTPT $1 \times 1$ ordering; Step 9. Make on all $\widetilde{\chi_{\iota \jmath}}$ in cell of $(\imath, \jmath)^{t h}$ that are given by FTPT; Step 10. Resulted of IBFS $\widetilde{\varsigma_{\imath \jmath}}$ is $\widetilde{\chi_{\iota j}}$ and Eq. (1), respectively.

\section{REFERENCES}

[1] A. Ebrahimnejad, "A duality approach for solving bounded linear programming problems with fuzzy variables based on ranking functions and its application in bounded transportation problems, " Int. J. of System Sci. Iran. vol. 46, pp. 2048-2060, 2015.

[2] A. Hatami-Marbini and M. Tavana, "An extension of the linear programming method with fuzzy parameters," Int. J. of Math. in Operational Research. Belgium, vol. 03, pp. 44-55, 2011.

[3] S. Saati, A. Hatami-Marbini, M. Tavana and E. Hajiahkondi, "A two fold linear programming model with fuzzy data," Int. J. of Fuzzy Syst. Appl.. Belgium, vol. 2, pp. 1-12, 2012.

[4] G. Zhang and H. Zuo, Research on multi-objective linear programming with fuzzy coefficients constraints," Int. J of Machine Learning and Cyber-netics . China, vol. 5, pp. 403-412, 2014.

[5] A. N. Gani and K. A. Razak, "Two stage fuzzy transportation problem," J. of Phys. Sci. India, vol. 10, pp. 63-69, 2006.

[6] D.S. Dinagar and K. Palanivel, "The transportation problem in fuzzy environment," Int. J. Algorithm Math. Comp. India, vol. 2, pp. 65-67, 2009.

[7] P. Pandian and G. Natarajan, "A new algorithm for finding a fuzzy optimal solutionfor fuzzy transportation problems," Appl. Math. Sci. India, vol. 4, pp. 79-90, 2010.

[8] A Chakraborty and M. Chakraborty, "Cost-time minimization in a transport problem with fuzzy parameters: a case study," J. of Transport Systems Engineering and Information Technology. India, vol. 10, pp. $53-63,2010$
Algorithm 4 Step 1. The IBFS obtained of FTP using new least cost method; Step 2. Introduce $\kappa_{\imath}$ and $\iota_{j}$ as variable convenient for every $\imath^{t h}$ and $j^{\text {th }}$, respectively. In front of $\imath^{\text {th }}$, write $\kappa_{\imath}$ in row and $\iota_{j}$ at the under of $j^{\text {th }}$ in column. The simplify calculation, with $j^{\text {th }}=0$ is maximum number of allocations row; Step 3. Determine $\kappa_{\imath}$ and $\iota_{j}$ others values by using $\varsigma_{\imath \jmath}=\kappa_{\imath}+\iota_{\jmath}$ for base of cell, then determine $\theta_{\imath \jmath}=\varsigma_{\imath \jmath}-\left(\kappa_{\imath}+\iota_{\jmath}\right)$ of non-base of cells. Next, will come two possibilities as follow; $(i) \theta_{\imath \jmath} \geq 0 \forall \imath \jmath$. So, the resulted of IBFS is done i.e fuzzy optimal solution has been satisfied; (ii) Otherwise, $\exists \theta_{\imath \jmath}<0$ So, obtained IBFS do not finished yet i.e fuzzy optimal solution is not optimal. Therefore, the getting of fuzzy optimal solution is chosen a cell of $(\imath, \jmath)^{t h}$ that $\theta_{\imath \jmath}$ in which rank is smallest negative of $\theta_{\imath \jmath}$, then make a horizontal and vertical path closed that starts from unchosen base of cell of $(\imath, \jmath)^{t h}$. The path can only replace to angle on base of cell $(\imath, \jmath)^{t h}$ and the path is chosen path must pass through base and non-base cell of $(\imath, \jmath)^{t h}$; Step 4 . Give sign $(+)$ and $(-)$ for closed loop started with $(+)$ for chosen nonbase cells. After that, determine fuzzy quantity on cells with signs $(+)$ and $(-)$. Consequently, will be obtained new FTPT. Step 5. Repeat of steps 2, 3 and 4 for new FTPT until $\theta_{\imath \jmath} \geq 0$ $\forall \imath$; Step 6 . Determine the value of fuzzy optimal solution or objective function by using Eq. (1).

[9] H. Basirzadeh, "An approach for solving fuzzy transport problem," Appl. Math. Sci. Iran, vol. 5, pp. 1549-1566, 2011.

[10] A. Kaur and A. Kumar, "A new method for solving fuzzy transport problems using ranking function," Appl. Math. Model. Iran, vol. 35, pp. $5652-5661,2011$.

[11] A. Kaur and A. Kumar, "A new approach for solving fuzzy transport problems using generalized trapezoid fuzzy numbers," Appl. Soft Comput. Iran, vol. 12, pp. 1201-1213, 2012.

[12] M. R. Fegade, V. A. Jadhav, and A. A. Muley, "Solving Fuzzy Transport Problem Using Zero Suffix and Robust Ranking Methodology," IOSR J. of Engineering (IOSRJEN). India, vol. 2, pp. 36-39, 2012.

[13] S. Mohanaselvi and K. Ganesan, "Fuzzy Optimal Solution to Fuzzy Transport Problem: A New Approach," Int. J. on Computer Science and Engineering (IJCSE). India, vol. 4, pp. 367-375, 2012.

[14] A. E. Samuel and M. Venkatachalapathy, "A New Dual Based Approach for the Unbalanced Fuzzy Transport Problem," Appl. Math. Sci. India, vol. 6, pp. 4443-4453, 2012.

[15] M. Shanmugasundari and K. Ganesan, "A novel approach for the fuzzy optimal solution of fuzzy transportation problem," Int. J. Eng. Research Appl. India, vol. 3, pp.1416-1421, 2013.

[16] C. Sudhagar and K. Ganesan, "A fuzzy approach to transport optimization problem," Optim. Eng. New York, vol. 17, pp. 965-980, 2013.

[17] F. A. Giarcarlo, C. X. C. A. Barbara and E. W. Volmir, "New Methodology to Find Initial Solution for Transport Problems: a Case Study with Fuzzy Parameter," Appl. Math. Sci. New York, vol..9, pp. 915-927, 2015.

[18] M. Sam'an, Farikhin S. Hariyanto and B. Surarso, "Optimal solution of full fuzzy transportation problems using total integral ranking," IOP Conf. Series: Journal of Physics: Conf. Series. Indonesia, vol. 983. pp. 1-7, August 2018. [International Conference on Mathematics, Science and Education 2017 (ICMSE2017)].

[19] M. Sam'an, Farikhin, B. Suraso and S. Zaki, "A modified algorithm for full fuzzy transportation problem with simple additive weighting," IEEE of Conf. Series. Indonesia, April, 2018. [2018 International Conference on Information and Communications Technology (ICOIACT)].

[20] T.S. Liou, and M.J Wang, "Ranking fuzzy number with integral values," Fuzzy Sets and Syst. New York, vol. 50, pp. 247-255, 1992.

[21] A. Ebrahimnejad, "A simplified new approach for solving fuzzy transport problems with generalized trapezoid fuzzy numbers," Appl. Soft Comput. Iran, vol. 19, pp. 171-176, 2014. 